\title{
Tai Chi Chuan in postsurgical non-small cell lung cancer patients: study protocol for a randomized controlled trial
}

\author{
Hong Pan ${ }^{1,2+}$, Yingxia Pei ${ }^{1 \dagger}$, Bingxue Li ${ }^{1,3}$, Yi Wang ${ }^{1}$, Jie Liü ${ }^{* *}$ and Hongsheng Lin ${ }^{* *}$
}

\begin{abstract}
Background: Impairment of exercise capacity remains a common adverse effect of non-small cell lung cancer (NSCLC) survivors after surgery. Previous research has suggested that Tai Chi Chuan (TCC) offers an exercise capacity benefit in several types of cancers. This is a randomized trial to investigate the efficacy and safety of TCC in postoperative NSCLC patients over an observation period of 3 months and a 9-month follow-up.

Methods/design: Using a prospective, one center and randomized design, 120 subjects with histologically confirmed stage I-IIIA NSCLC following complete surgical resection will potentially be eligible for this trial. Following baseline assessments, eligible participants will be randomly assigned to one of two conditions: (1) TCC training, or (2) placebo control. The training sessions for both groups will last $60 \mathrm{~min}$ and take place three times a week for 3 months. The sessions will be supervised with target intensity of $60-80 \%$ of work capacity, dyspnea, and heart rate management. The primary study endpoint is peak oxygen consumption $\left(\mathrm{VO}_{2 \text { peak }}\right)$, and the secondary endpoints include: 6-min walk distance (6MWD), health-related quality of life (HRQoL), lung function, immunity function, and the state of depression and anxiety. All endpoints will be assessed at the baseline and postintervention (3 months). A follow-up period of 9 months will be included. The main time points for the evaluation of clinical efficacy and safety will be months 3, 6, 9, and 12 after enrollment.

Discussion: This study will assess the effect of group TCC in postsurgery NSCLC survivors on $\mathrm{VO}_{2 \text { peak, }}$ lung function, and other aspects. The results of this study will eventually provide clinical proof of the application of TCC as one kind of exercise training for patients across the entire NSCLC continuum, as well as information on the safety and feasibility of exercise.
\end{abstract}

Trial Registration: Chinese Clinical Trial Registry: ChiCTR-IOR-15006548. Registered on 12 June 2015.

Keywords: Tai Chi Chuan, Exercise capacity, Non-small cell lung cancer, Postoperation

\section{Background}

Lung cancer is a prevalent issue worldwide. Data from 2011 indicated that lung cancer, comprising $18.6 \%$ of the total new cases and $13.6 \%$ of the total deaths, is the most frequently diagnosed cancer in males and the leading cause of cancer death for each sex in both economically developed and developing countries [1]. With a 5-year survival of $14 \%$, it was estimated that about 159,260 Americans would die from lung cancer in 2014 [2]. Lung

\footnotetext{
*Correspondence: dr.liujie@163.com; dr_linhs@163.com

${ }^{\dagger}$ Equal contributors

'Department of Oncology, Guang'anmen Hospital, China Academy of

Chinese Medical Sciences, Beijing 100053, China

Full list of author information is available at the end of the article
}

cancer is histologically categorized into small cell lung cancer (SCLC) and non-small cell lung cancer (NSCLC) (approximately $85 \%$ of those diagnosed with lung cancer), and the survival of patients with the latter is much higher than those diagnosed with SCLC [3].

Surgical removal remains the best curative option for patients with early-stage NSCLC and for appropriately selected patients with locally advanced disease. Approximately 26,000 individuals per year in the United States will survive more than 5 years after initial diagnosis of operable disease [4]. With the government's focus on the early detection of lung cancer screening, an increasing number of patients with early-stage disease were 
detected, thus elevating the number of curable and surgical resection cases [5]. Despite the improvement in surgical techniques and the survival rate, surgery is still associated with significantly reduced exercise capacity, which in turn is associated with increased morbidity $[6,7]$ along with functional limitations, decreased health-related quality of life (HRQoL), and psychiatric issues [8].

Clinicians are paying more and more attention to improving overall physical and emotional function as well as longevity. A growing interest has arisen in the use of non-pharmacological interventions such as exercise, which has been identified as a safe and effective way to improve aerobic capacity, strength, body composition, and HRQoL, as well as to reduce fatigue, emotional distress, and lymphedema symptoms in diverse cancers [9-14]. To our knowledge, preliminary data suggest that exercise intervention compared with usual care is associated with improved exercise capacity for postsurgery patients [15]. Nevertheless, the results have been conflicting and the optimal program is still to be determined, such as the type of exercises [16].

Tai Chi Chuan (TCC) is a traditional Chinese exercise oriented in thousands of years of ancient history. It combines meditation with slow and gentle movements, as well as deep breathing to move vital qi throughout the body. It is considered as a complex, multicomponent intervention that involves physical, psychosocial, emotional, spiritual, and behavioral elements [17]. The results of a systematic review including 1868 participants suggested that TCC has a positive effect on the majority of outcomes of exercise capacity including peak oxygen consumption $\left(\mathrm{VO}_{2 \text { peak }}\right)$ [18]. Furthermore, a recent meta-analysis that pooled the effect of 12 controlled trials examining the efficacy of TCC to improve the cancer-specific HRQoL reported that the weighted mean difference was 7.99 (95\% confidence interval (CI) 4.07-11.91; $Z$ score $=4.00, p<0.0001$ ) [19]. In addition, compared with conventional exercises, research among 61,477 adults revealed TCC as the most common forms of exercise among Chinese adults and is related to reduced cancer-caused mortality [20]. Meanwhile, as a mind-body technique, TCC has been found to better lower mental disorder and fall rate in cancer survivors relative to conventional therapeutic exercise [21]. Given the clinical benefits of TCC, none of these studies evaluated the influence of TCC among postoperative NSCLC patients.

In light of this evidence, we designed a single-blind randomized controlled trial to evaluate the efficacy and feasibility of TCC on postoperative NSCLC patients. We hypothesize that TCC may serve as a safe exercise intervention that offers potential benefit among postsurgical NSCLC survivors.

\section{Methods}

\section{General objective}

To investigate the efficacy and feasibility of TCC on postoperative NSCLC patients with the aim of confirming the effectiveness and safety of TCC.

\section{Study design and procedures}

The study will be a prospective, single blind, parallel group, randomized controlled trial. We will recruit and randomize 120 histologically confirmed stage I-IIIA postsurgical NSCLC patients 1:1 into two groups: 1) TCC training; or 2) placebo control. The study period will be 12 months including a 3-month supervised exercise training course and a 9-month follow-up course with the first and secondary outcomes measured at baseline, and at 3, 9, and 12 months, along with other assessments. The procedure is shown in Fig. 1. All patient visits, exercise, and assessment will take place in Guang'an men Hospital, China Academy of Chinese Medical Sciences, Beijing, China. This report will be compiled in line with the SPIRIT (Standard Protocol Items: Recommendations for Interventional Trials) 2013 Statement "Defining Standard Protocol Items for Clinical Trials" [22]. Additional file 1 shows this in more detail.

\section{Participants}

This study will be conducted in the Department of Oncology, Guang'an men Hospital, China Academy of Chinese Medical Sciences. Each member of staff of the study team will be able to enroll participants. Inclusion criteria will be as follows: 1) histologically diagnosed with Stage I-IIIA NSCLC according to the 7th edition cancer staging manual of the American Joint Committee on Cancer [23] and had received surgical therapy; 2) had survived no more than 6 months since surgery; 3) Karnofsky performance status of at least $70 \%$ at study entry; 4) estimated life expectancy $\geq 6$ months; and 5) aged between 18 and 65 years. All study subjects will meet all five inclusion criteria.

Exclusion criteria will be as follows: 1) subjects with serious systemic diseases, such as cerebrovascular, liver, kidney, hematopoietic system, and psychiatric diseases; 2) unable to participate in an exercise training because of either bone or joint diseases; 3 ) patients who are in other clinical trials; and 4) unable to understand Chinese. Every enrolled patient will sign an informed consent (seen in Additional file 2) before taking part in the later project procedures.

Measures to guarantee adequate participant enrolment will include: 1) a free rehabilitation and exercise training program; 2) the offer of priority access to specialist consultation; and 3) additional general guidance and disease consultation. 


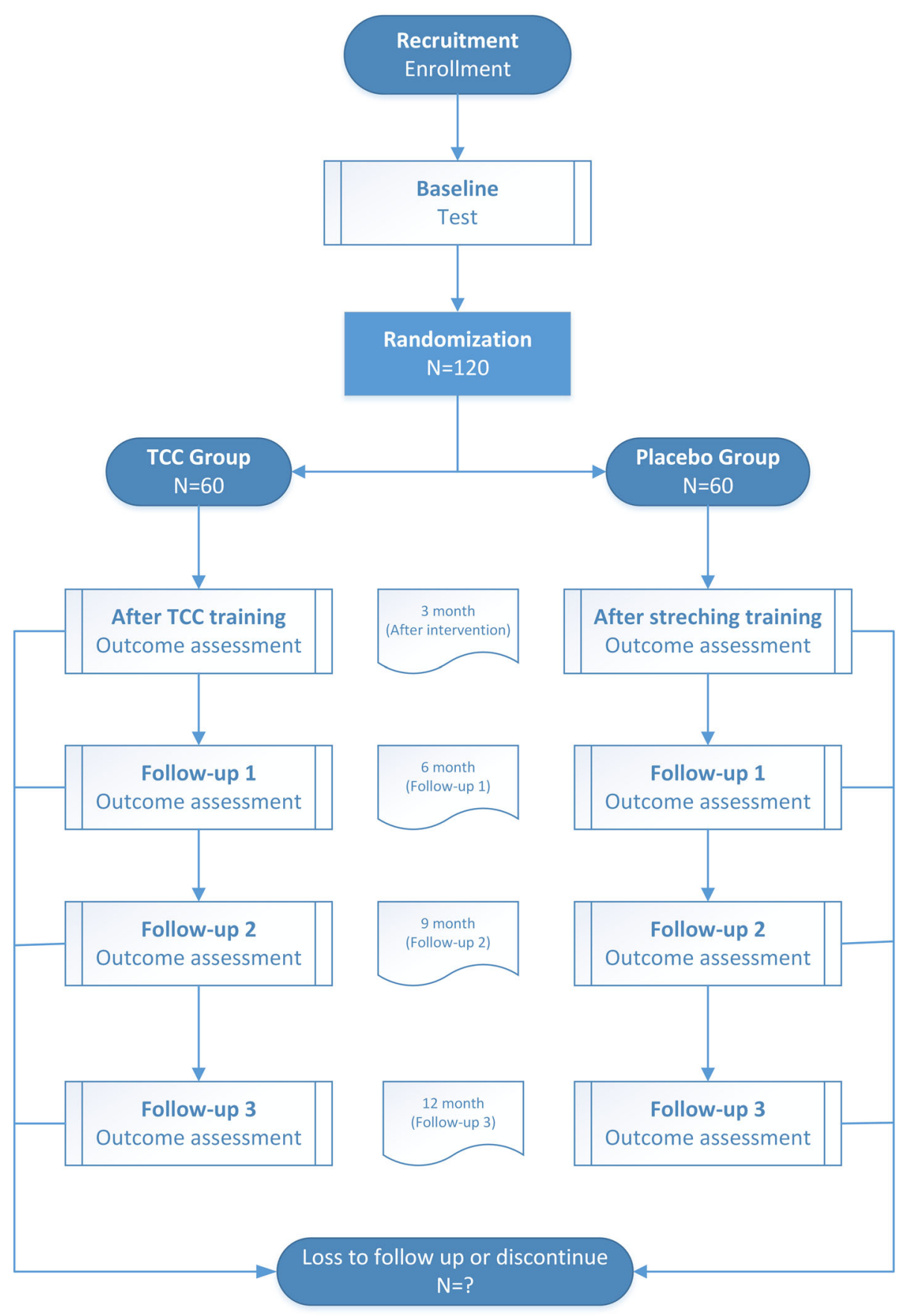

Fig. 1 Flow diagram. TCC Tai Chi Chuan

\section{Randomization and blinding}

After completing baseline testing, each participant will be randomly allocated in a 1:1 ratio to either the TCC group or the placebo group. Each participant will learn of their group assignment by receiving a sequentially numbered and sealed opaque envelope from the project director and opening the envelope that contains the group assignment randomly assigned to their sequence number as randomly generated by the computer. Measurement technicians who are responsible for data collection and coding data are blinded to participant group assignment. In addition, all study personnel conducting the study assessments at baseline and postintervention will be blinded to treatment assignment for the duration of the study. 


\section{Study interventions}

Participants in each group will attend a supervised 1-h exercise session three times per week, starting 4 weeks after surgery at the earliest. A senior exercise physiologist will conduct the exercise session. Each session includes 10 min low-intensity warming up (50-60\% of maximum heart rate) and 5 min cooling down at the beginning and end of the course, respectively. All interventions will be tailored to each participant following the principles of aerobic or resistance training prescription guidelines for adults as recommended by the American College of Sports Medicine (ACSM) [24]. The Borg rating of perceived exertion (RPE) and blood pressure will be assessed prior to and after the exercise, with heart rate and $\mathrm{O}_{2}$ saturation monitored continuously throughout.

\section{TCC training}

The TCC training group will learn to perform 24 standard TCC movements and breathing techniques taught by a professional TCC master with more than 20 years of teaching experience. The instructor explains the theory behind TCC and provides printed materials about TCC when the patient is placed in this group. In each session, participants practice TCC under the master's instruction. Each session includes a 10-min warm-up about self-massage, breathing techniques, and relaxation in TCC, $45 \mathrm{~min}$ exercise training and $5 \mathrm{~min}$ cooling down. Throughout the intervention period, participants are required to practice TCC for 20 min every 4 days at home. After 3 months, participants are encouraged to practice TCC using an instructional DVD and keep record of activities until the follow-up visit at month 12 .

\section{Placebo control}

Participants in the placebo control group will attend a supervised stretching program of the same frequency, duration, and length as the TCC group. The stretching program will be prescribed according to ASCM guidelines for people aiming to increasing whole-body flexibility. Stretches will be performed from a seated position in order to minimize weight-bearing forces. The stretching will also be limited in duration to $45 \mathrm{~min}$, alternating between lower and upper body muscle groups in combination with warm-up and cooling down under the supervision of a professional physiologist. Throughout the intervention period, participants will be asked to stretch for 20 min every 4 days at home. After 3 months, participants will be encouraged to do stretching until the follow-up visit at month 12 and keep a record.

\section{Adherence considerations}

Participants will be encouraged to accomplish the routine exercise over the 3 months. Several strategies will be employed to maximize adherence including individualized attention at the intervention sessions and telephone calls following missed sessions. In addition, participants who miss a session will be asked to attend a make-up class. Furthermore, we set the same classes in the morning and afternoon for patients to choose from, which promotes attendance by improving accessibility. Thirdly, all the participants will be asked to complete daily logs about the amount of time they practiced TCC or stretches. Finally, the study staff will also meet on a weekly basis to review each participant's adherence.

\section{Study endpoints and assessments Primary endpoint}

$\mathrm{VO}_{\text {2peak }} \mathrm{VO}_{2 \text { peak }}$ will be evaluated using a physiciansupervised incremental cycle ergometer test with 12-lead electrocardiogram (ECG) monitoring ( $\mathrm{Mac}^{\circ}$ 5000, GE Healthcare) under the supervision of a physiologist blinded to the patient's randomization group. According to prior studies by Jones et al. [25, 26], all the subjects will pedal at $20 \mathrm{~W}$ for $1 \mathrm{~min}$, and increase by $5 \mathrm{~W}$ every minute until exhaustion or a symptom-limited $\mathrm{VO}_{2 \text { peak }}$ is achieved. Exercise will be terminated if any ECG abnormalities are observed.

\section{Secondary endpoints}

The secondary endpoints of this trial will be the 6-min walk distance (6MWD), lung function, patient-reported outcomes, and immunity function.

6MWD 6MWD is a useful measure of functional capacity, which has been widely used for postoperative evaluation and for measuring the response to therapeutic interventions for pulmonary disease. The test will be performed according to current guidelines [27] in a 30-m corridor. Patients will be instructed to walk at their fastest pace and cover the longest possible distance in $6 \mathrm{~min}$; pauses are allowed if needed. Waiting at least $1 \mathrm{~h}$ before a second test, the highest 6MWD is reported. Measurement of peripheral oxygen saturation and heart rate will be performed before and immediately after the test. Participants were asked to rate their post-test dyspnea and fatigue level using the Borg scale (rate 6-20) [28], with a higher rating corresponding to higher dyspnea or fatigue level.

Lung function Lung function was found to be significantly associated with postoperative mortality in NSCLC, especially the percentage of forced expiratory volume in $1 \mathrm{~s}$ (FEV1) $[29,30]$. This parameter will be evaluated using a calibrated spirometer (Spirovit SP-2; Schiller, Swizerland). FEV1 and forced vital capacity (FVC) will be measured, and FEV1/FVC will be calculated and compared to reference values [31]. The 
patients will be measured three times and the best result will be recorded.

Patient-reported outcomes Patient-reported outcomes will include HRQoL, fatigue, dyspnea, depression, and anxiety. The 42-item Functional Assessment of Cancer Therapy-Lung (FACT-L) scale, which contains four subscales for physical (seven items), functional (seven items), emotional (six items), and social/family (seven items) well-being, plus a lung cancer-specific subscale (15 items), will be used to assess patient symptoms and HRQoL [32]. The 13-item FACT-fatigue scale will be used as the assessment of fatigue [33]. The Cancer Dyspnea Scale (CDS), which has been demonstrated to be a valid and feasible scale for assessing cancer-related dyspnea among NSCLC patients [34, 35], will be used to evaluate dyspnea [36]. Finally, depression and anxiety will be assessed using the Self-rating Depression Scale (SDS) and the Self-rating Anxiety Scale (SAS). All the above instruments have been found to be easy to administer, widely used, and reliable in prior research in lung cancer patients.

Immunity function Immunity function will be tested by using peripheral blood samples collected at 7:00 am at baseline, at completion of the 3-month study, and at 9 months follow-up. Flow cytometry (Beckman Coulter EPICS XL; Beckman Coulter, Inc., Brea, CA, USA) will be used for the analysis within $24 \mathrm{~h}$ of collection of the blood samples. The percentage of $\mathrm{T}$ lymphocytes, $\mathrm{CD} 4^{+}$ and $\mathrm{CD}^{+} \mathrm{T}$ lymphocytes, and the ratio of $\mathrm{T}$ helper/T suppresser cells will be used for the assessment.

\section{Tracking and monitoring of adverse events}

Several methods will be used to assure the tracking and monitoring of adverse events: 1) all patients will be under the supervision of an oncologist and every single adverse event will be recorded on a case report form (CRF); 2) at the beginning of the training intervention, the physiologist will discuss the potential side-effects of the exercise, and all episodes occuring during the class will be recorded on the CRF; 3) during the exercise course and assessment, the blood pressure, heart rate, and $\mathrm{SpO}_{2}$ will be monitored in case of any negative side-effect; 4) any severe adverse event will be reported to the monitor of the trial and Guang'an Men Hospital within $24 \mathrm{~h}$.

The time schedule of the study, including enrollment, interventions, assessments, and visits for participants, is shown in Fig. 2.

\section{Data management and monitoring}

A specialized clinical research associate (CRA) will be responsible for checking the ranges for data values. Double data entry will be conducted to promote data quality. Operations and data will be audited at the end of the trial by the state administration of traditional Chinese medicine, which is the department that oversees the project. Clinical Research Organizations (CRO), which are independent from investigators and the sponsor, will monitor the data during every participant visit.

\section{Statistical analysis}

This randomized trial will recruit 120 subjects with postsurgical NSCLC. Unpaired $t$ tests will be used to analysis the primary and secondary endpoints by comparing the TCC to the placebo group regarding mean improvement. We assume that improvement in $\mathrm{VO}_{2 \text { peak }}$ in the TCC group will be $22.5 \%$, while it will be $5 \%$ in the placebo group, as reported previously [15]; thus, a minimum of 55 patients per group will be required. Accounting for a $10 \%$ dropout rate, the inclusion of 60 patients per group results in a total of 120 patients.

The principal analysis of the study endpoints will employ the intention-to-treat (ITT) approach. The ITT analysis will include all randomized participants in the random allocation. The intervention group assignment will not be altered based on the participants' adherence to the randomly allocated study arm. Patients who are lost to follow-up will be included in all primary and secondary analyses by assuming zero change across time. For the primary analysis, a multiple regression model will be used to regress change in $\mathrm{VO}_{2 \text { peak }}$ on study group, the baseline value of the endpoint, and other pertinent baseline variables that may influence change in the study endpoints (e.g., co-morbid conditions/medications, self-reported exercise history, age).

All statistical analysis will be conducted using SPSS 17.0 statistics software. The data of all the endpoints are quantitative data. The mean \pm standard deviation will be used to describe symmetric data validated by distribution pattern and homogeneity of variance, otherwise the interquartile range (IQR) will be used. If the data are symmetric after being validated by distribution pattern and homogeneity of variance, a $t$ test will be used to compare the differences between the quantitative indices between the groups, otherwise the Wilcoxon rank-sum test will be used. For each endpoint, the overall alpha level will be controlled at a one-sided 0.05 by using Holm's procedure [37]. The collected data will be examined, and the researchers will be supervised to control bias.

\section{Discussion}

Surgical removal is the best curative option for patients with early-stage NSCLC and for appropriately selected patients with locally advanced disease. Increasing numbers of patients are eligible for receiving surgical intervention [5]. Despite the possibility of a cure, NSCLC survivors with lung resection surgery are often 


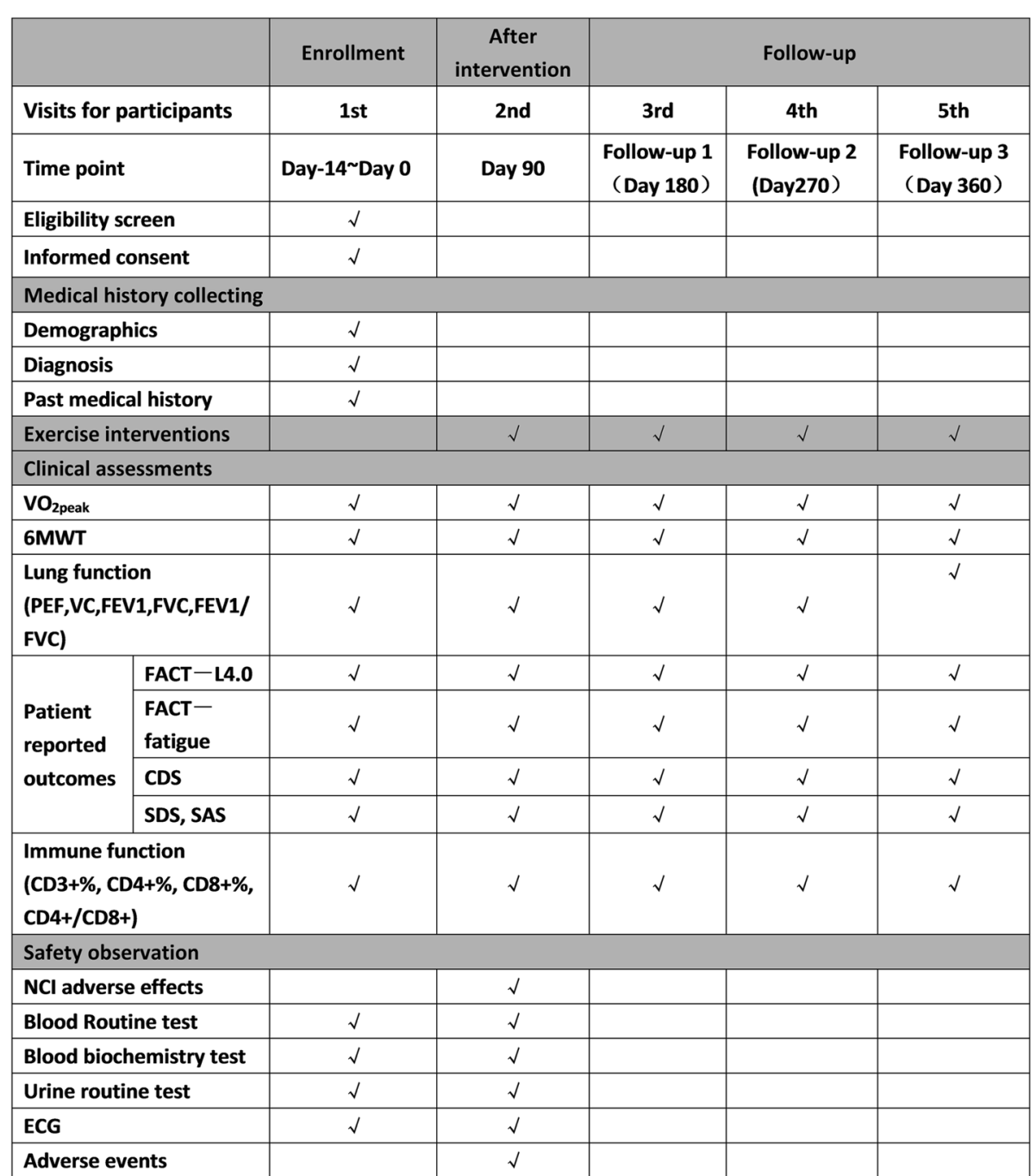

Fig. 2 Time schedule of enrollment, interventions, and assessments, as well as visits for participants. 6MWT 6-min walk test, CD cluster of differentiation, CDS Cancer Dyspnea Scale, ECG electrocardiograph, FACT Functional Assessment of Cancer Therapy, FEV1 forced expiratory volume in $1 \mathrm{~s}$, FVC forced vital capacity, NCI National Cancer Institute, PEF parameter peak flow, SAS Self-rating Anxiety Scale, SDS Self-rating Depression Scale, $V O_{2 p e a k}$ Peak oxygen consumption

deconditioned and may have varying degrees of impairment of exercise capacity [38] and HRQoL [39] due to the unique pathophysiology of NSCLC, conventional therapeutic strategy, and physical inactivity. Additionally, studies conclude that operable NSCLC patients are at a higher risk of developing emotional distress than other diagnosis groups [40]. Surgically treated NSCLC patients are reported to suffer from persistent physical functional impairment lasting up to 12 months after surgery or longer [41]. Recently, epidemiologic studies and findings suggest that regular exercise can decrease the cancerrelated mortality and all-cause mortality among cancer survivors [42], and has been identified as a successful intervention to improve physical and psychological health in some cancer populations (mainly breast cancer) [43]. In light of the increase in the diagnosis of people with curable lung cancer, with the likelihood of an anticipated changing landscape of cancer care, exercise intervention for this population needs to be explored urgently.

TCC, a traditional Chinese exercise, has gained a lot of attention in clinical and research applications as a rehabilitation method for cancer survivors. Recent systematic reviews have demonstrated that TCC may have enormous potential as an effective and feasible rehabilitation intervention to improve tolerance of exercise capacity, symptoms, and immunity function for cancer survivors [18, 19]. In addition, TCC is one of the most common forms of exercise among Chinese adults [20] indicating that there is a large potentially eligible population that would be able to be recruited in to the trial. 
The main outcome of the present trial is exercise capacity. The gold standard for measurement of exercise capacity is a direct measure of $\mathrm{VO}_{2 \text { Peak }}$; other testing methods include the $6 \mathrm{MWD}$, shuttle walk test, and stairclimbing test. Among these, $\mathrm{VO}_{2 \text { peak }}$ and $6 \mathrm{MWD}$ are the two most frequently used (52\% and 16\%) [44]. Furthermore, $\mathrm{VO}_{2 \text { Peak }}$ is an independent predictor of all-cause pulmonary morbidity $[45,46]$. Brunelli and colleagues found that a $\mathrm{VO}_{2 \text { Peak }}$ of no less than $20 \mathrm{~mL} / \mathrm{kg} / \mathrm{min}$ is a safe cutoff value, and no mortality was observed above this threshold for major lung resection [47]. Consistent with this, the findings of Jones et al. suggest that higher $\mathrm{VO}_{2 \text { peak }}$ was associated with a statistically significant $21 \%$ to $24 \%$ reduction in the risk of mortality, and a $\mathrm{VO}_{2 \text { peak }}$ of $<14 \mathrm{~mL} / \mathrm{kg} / \mathrm{min}$ may identify patients with poor prognosis [48]. Together, this indicates that $\mathrm{VO}_{\text {2peak }}$ is an emerging evaluating parameter of exercise training in patients with NSCLC. Specifically, research has shown TCC to be beneficial to the $\mathrm{VO}_{2 \text { peak }}$ of middle-aged subjects $[49,50]$. Despite the wealth of evidence, research has seldom explored the efficacy of TCC against exercise capacity or $\mathrm{VO}_{2 \text { peak }}$. Thus, the primary outcome of this trial will be VO2peak. 6MWD and lung function will be set as simple and alternative elements to comprehensively assess the exercise capacity of this specific population.

Patients with operable NSCLC also experience fatigue and dyspnea, and are at risk for developing psychosocial problems, reduced quality of life as well as immune function. And, existing evidence have suggested that TCC has a promising function on enhancing proliferative and cytolytic activities of peripheral blood mononuclear cells (PBMCs), as well as increasing the ratio of $\mathrm{T}$ helper/ $\mathrm{T}$ suppressor cells and mediating Th1/Th2 T-helper cell balance in postsurgical NSCLC patients, thus improving the immunity function of the patients [51,52]. Therefore, our study also takes above symptom and immunity parameters into consideration to comprehensively evaluate the clinical benefit of TCC as an exercise intervention.

To enable TCC intervention to be integrated into clinical practice, it is important to determine the optimal design of TCC intervention that will be feasible, acceptable, and positively affect outcomes in a healthcare setting as rehabilitation in postsurgical patients with NSCLC in a randomized clinical trial. To our knowledge there are currently no clinical trial focusing on TCC intervention for surgically treated NSCLC patients. Therefore, the results of this study will offer an overview of the possible effects of TCC and address many critical unknown questions in order to set the stage for more clinical trials using traditional Chinese exercises such as Qigong and Baduanjin. In the long term, we are looking forward to making a contribution to the establishment of exercise interventions as a non-pharmacologic therapeutic guideline for NSCLC patients.

While the trial will potentially significantly contribute to these fields of research, potential limitations of the study should also be recognized. One difficulty encountered in the design of the trial was selecting an appropriate control condition. There were several options for control participants including: 1) strength training; 2) conventional aerobic exercise; and 3) stretching placebo. Although the first two of these have shown clinical benefit for NSCLC, neither of them was selected. Strength training was not selected because it is too vigorous and unacceptable for NSCLC based on our prior oral pilot questionnaire. Furthermore, the target of the trial is to explore the clinical efficacy and feasibility of TCC, rather than the comparison between TCC and aerobic exercise; thus, stretching as a more tolerable and safe control was selected.

\section{Trial status}

The study will end in 2017. To date, all 120 patients have been recruited.

\section{Additional file}

Additional file 1: SPIRIT 2013 Checklist: recommended items to address in a clinical trial protocol and related documents. (DOC $128 \mathrm{~kb}$ )

Additional file 2: Informed Consent form - Informed notice page (Chinese version). (DOC $44 \mathrm{~kb}$ )

\section{Abbreviations}

6MWD: Six-minute walk distance; ACSM: American College of Sports Medicine; CDS: Cancer Dyspnea Scale; Cl: Confidence interval; CRA: Clinical Research Associate; CRF: Case report form; CRO: Clinical Research

Organization; ECG: Electrocardiogram; FACT-L: Functional Assessment of Cancer Therapy - Lung; FEV1: Forced expiratory volume in 1 second; FVC: Forced vital capacity; HRQoL: Health-related quality of life; IQR: Interquartile range; ITT: Intention-to-treat; NSCLC: Non-small cell lung cancer; PBMC: Peripheral blood mononuclear cell; RPE: Rating of perceived exertion; SAS: Self-rating Anxiety Scale; SCLC: Small cell lung cancer;

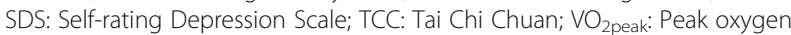
consumption

\section{Acknowledgements}

The authors thank those who have participated in the trial. They are also grateful to the staff of the participating center

\section{Funding}

This study is funded by the industry-specific project: The demonstration and promotion of TCM clinical guidelines for Lung cancer (no. 201307006).

Availability of data and materials

To date, no new data have been created during this study.

\section{Authors' contributions}

HP is the first author who thought of the concept and design, drafted the manuscript, and read and approved the final version. YP is the co-first author who contributed equally to this work. BL revised the manuscript and approved the final version. YW read and approved the final version. $J \mathrm{~L}$ and $\mathrm{HL}$ are the corresponding authors who conceived and designed the study, drafted the manuscript, and read and approved the final version. All authors read and approved the final manuscript. 


\section{Ethics approval and consent to participate}

The implementation of this project has been reviewed and approved by the Ethics Review Committee of Guang'anmen Hospital, China Academy of Chinese Medical Sciences. The Certificate number of the ethics review is 2014EC071-01. This trial was designed and will be implemented in accordance with the relevant provision on the protection of rights and interests of the subjects in the Declaration of Helsinki version 08 and the 'Biological Ethics Review Method Involving Humans' by the International medical science organization committee. Written informed consent (see Additional file 2) will be obtained from all participants prior to initiation of any study procedure by the PI or clinical research coordinators prior to enrollment, and subjects will be given adequate time to declare if they wish to participate in this study before signing the consent form. Study participation is voluntary and can be cancelled at any time without provision of reasons and without negative consequences for their future medical care.

The PI and clinical research coordinators will have full access to the study dataset. All research-related paper files will be stored in locked cabinets in the Cancer Institute of China Academy of Chinese Medical Sciences in Guang'anmen Hospital, and all electronic files will be stored on a secure research server. No personal identifiable information will be shared outside of the research server.

The full study protocol is made public and available in Trials. At study completion, the PI and clinical research coordinators will prepare a manuscript for submission to a scientific journal to report study findings using de-identified study information. The dataset and statistical analysis will be published according to journal publication guidelines.

\section{Consent for publication}

Not applicable.

\section{Competing interests}

The authors declare that they have no competing interests.

\section{Publisher's Note}

Springer Nature remains neutral with regard to jurisdictional claims in published maps and institutional affiliations.

\section{Author details}

'Department of Oncology, Guang'anmen Hospital, China Academy of Chinese Medical Sciences, Beijing 100053, China. ${ }^{2}$ Department of Oncology, the first affiliated hospital of Zhejiang Chinese Medical Hospital, Zhejiang 310006, China. ${ }^{3}$ Clinical Medical College, Beijing University of Chinese Medicine, Beijing 100029, China.

Received: 24 March 2017 Accepted: 6 November 2017

\section{Published online: 04 January 2018}

\section{References}

1. Jemal A, Bray F, Center MM, Ferlay J, Ward E, Forman D. Global cancer statistics. CA Cancer J Clin. 2011;61:69-90.

2. Siegel R, Ma J, Zou Z, Jemal A. Cancer statistics. CA Cancer J Clin. 2014;64:9-29.

3. Sher T, Dy GK, Adjei AA. Small cell lung cancer. Mayo Clin Proc. 2008;83:355.

4. Peddle CJ, Jones LW, Eves ND, Reiman T, Sellar CM, Winton T, et al. Effects of presurgical exercise training on quality of life in patients undergoing lung resection for suspected malignancy: a pilot study. Cancer Nurs. 2009; 32:158-65.

5. Kenny PM, King MT, Viney RC, Boyer MJ, Pollicino CA, McLean JM, et al. Quality of life and survival in the 2 years after surgery for non small-cell lung cancer. J Clin Oncol. 2008;26:233-41.

6. Gulati M, Pandey DK, Arnsdorf MF, Lauderdale DS, Thisted RA, Wicklund RH, et al. Exercise capacity and the risk of death in women: the St James Women Take Heart Project. Circulation. 2003:108:1554-9.

7. Myers J, Prakash M, Froelicher V. Exercise capacity and mortality among men referred for exercise testing. N Engl J Med. 2002;346:793-801.

8. Handy JJR, Asaph JW, Skokan L, Reed CE, Koh S, Brooks G, et al. What happens to patients undergoing lung cancer surgery? Outcomes and quality of life before and after surgery. Chest. 2002;122:21-30.

9. Rogers LQ, Hopkins-Price P, Vicari S, Pamenter R, Courneya KS, Markwell S, et al. A randomized trial to increase physical activity in breast cancer survivors. Med Sci Sports Exerc. 2009;41:935-46.
10. Speck RM, Gross CR, Hormes JM, Ahmed RL, Lytle LA, Hwang WT, et al. Changes in the Body Image and Relationship Scale following a one-year strength training trial for breast cancer survivors with or at risk for lymphedema. Breast Cancer Res Treat. 2010;121:421-30.

11. Brocki B, Rodkjær L, Nekrasas V, Due K, Dethlefsen C, Andreasen J. Rehabilitation after lung cancer operation_a randomised controlled study. 20th ERS Annual Congress. 2010;36:331.

12. Arbane G, Tropman D, Jackson D, Garrod R. Evaluation of an early exercise intervention after thoracotomy for non-small cell lung cancer (NSCLC), effects on quality of life, muscle strength and exercise tolerance: randomised controlled trial. Lung Cancer. 2011;71:229-34.

13. Schmitz KH, Ahmed RL, Troxel A, Cheville A, Smith R, Lewis-Grant L, et al. Weight lifting in women with breast-cancer-related lymphedema. N Engl J Med. 2009;361:664-73

14. Allgayer H, Owen RW, Nair J, Spiegelhalder B, Streit J, Reichel C, et al. Short-term moderate exercise programs reduce oxidative DNA damage as determined by high-performance liquid chromatography-electrospray ionization-mass spectrometry in patients with colorectal carcinoma following primary treatment. Scand J Gastroenterol. 2008:43:971-8.

15. Edvardsen E, Skjønsberg OH, Holme I, Nordsletten L, Borchsenius F, Anderssen SA. High-intensity training following lung cancer surgery: a randomised controlled trial. Thorax. 2015;70:244-50.

16. Crandall K, Maguire R, Campbell A, Kearney N. Exercise intervention for patients surgically treated for non-small cell lung cancer (NSCLC): a systematic review. Surg Oncol. 2014;23:17-30.

17. Wayne PM, Kaptchuk TJ. Challenges inherent to tai chi research: part I —tai chi as a complex multicomponent intervention. J Altern Complement Med. 2008;14:95-102.

18. Zheng G, Li S, Huang M, Liu F, Tao J, Chen L. The effect of Tai Chi training on cardiorespiratory fitness in healthy adults: a systematic review and metaanalysis. PLoS One. 2015;10(2):e0117360. doi:10.1371/journal.pone.0117360.

19. Yingchun Z, Taizhen L, Huaan X, Meiling H, Andy SK. Health benefits of qigong or tai chi for cancer patients: a systematic review and metaanalyses. Complement Ther Med. 2014;22:173-86.

20. Chen M, He M, Min X. Different physical activity subtypes and risk of metabolic syndrome in middle-aged and older Chinese people. PLoS One. 2013;8:e53258.

21. Ruddy KJ, Stan DL, Bhagra A. Alternative exercise traditions in cancer rehabilitation. Phys Med Rehabil Clin N Am. 2017;28:181-92.

22. Chan AW, Tetzlaff JM, Altman DG, Laupacis A, Gotzsche PC, Krleza-Jeric K, et al. SPIRIT 2013 Statement: defining standard protocol items for clinical trials. Ann Intern Med. 2013;158:1-8.

23. Edge SB, Byrd DR, Compton CC, Fritz AG, Greene FL, Trotti A. AJCC cancer staging manual. 7th ed. New York: Springer; 2010.

24. American College of Sports Medicine Position Stand. The recommended quantity and quality of exercise for developing and maintaining cardiorespiratory and muscular fitness, and flexibility in healthy adults. Med Sci Sports Exerc. 1998;30:975-91.

25. Jones LW, Peddle CJ, Eves ND, Haykowsky MJ, Courneya KS, Mackey JR, et al. Effects of presurgical exercise training on cardiorespiratory fitness among patients undergoing thoracic surgery for malignant lung lesions. Cancer. 2007;110:590-8.

26. Jones LW, Eves ND, Hayowsky M, Joy AA, Douglas PS. Cardiorespiratory exercise testing in clinical oncology research: systematic review and practice recommendations. Lancet Oncol. 2008;9:757-65.

27. ATS Committee on Proficiency Standards for Clinical Pulmonary Function Laboratories. ATS statement: guidelines for the six-minute walk test. Am J Respir Crit Care Med. 2002;166(1):111-7.

28. Borg GA. Perceived exertion. Exerc Sport Sci Rev. 1974;2:131-53.

29. Powell HA, Tata $L$, Baldwin DR, et al. Early mortality after surgical resection for lung cancer: an analysis of the English national lung cancer audit. Thorax. 2013;68:826-34.

30. Friedel G, Fritz P, Goletz S. Postoperative survival of lung cancer patients: are there predictors beyond TNM? Anticancer Res. 2013;33:1609-19.

31. Quanjer PH, Tammeling GJ, Cotes JE, Pedersen OF, Peslin R, Yernault JC. Lung volumes and forced ventilatory flows. Report working party standardization of lung function tests, European community for steel and coal. Official statement of the European respiratory society. Eur Respir J Suppl. 1993;16:5-40.

32. Cella DF, Bonomi AE, Lloyd SR, Tulsky DS, Kaplan E, Bonomi P. Reliability and validity of the Functional Assessment of Cancer Therapy-Lung (FACT-L) quality of life instrument. Lung Cancer. 1995;12:199-220. 
33. Cella D. The Functional Assessment of Cancer Therapy-Anemia (FACT- An) Scale: a new tool for the assessment of outcomes in cancer anemia and fatigue. Semin Hematol. 1997;34:13-9.

34. Cesario A, Ferri L, Galetta D, Pasqua F, Bonassi S, Clini E, et al. Post-operative respiratory rehabilitation after lung resection for non-small cell lung cancer. Lung Cancer. 2007:57:118-9.

35. Bredin M, Corner J, Krishnasamy M, Plant H, Bailey C, A'Hern R. Multicentre randomised controlled trial of nursing intervention for breathlessness in patients with lung cancer. BMJ. 1999;318:901-4.

36. Tanaka K, Akechi T, Okuyama T, Nishiwaki Y, Uchitomi Y. Development and validation of the Cancer Dyspnoea Scale: a multidimensional, brief, self-rating scale. Br J Cancer. 2000;82:800-5.

37. Holm S. A simple sequentially rejective multiple test procedure. Scand J Stat. 1979;6:65-70.

38. Larsen KR, Svendsen UG, Milman N, Brenoe J, Petersen BN. Cardiopulmonary function at rest and during exercise after resection for bronchial carcinoma. Ann Thorac Surg. 1997;64:960-4.

39. Strasser B, Steindorf K, Wiskemann J, Ulrich CM. Impact of resistance training in cancer survivors: a meta-analysis. Med Sci Sports Exerc. 2013;45:2080-2090

40. Zabora J, BrintzenhofeSzoc K, Curbow B, Hooker C, Piantadosi S. The prevalence of psychological distress by cancer site. Psychooncology. 2001;10:19-28.

41. Schulte T, Schniewind B, Dohrmann $P$, et al. The extent of lung parenchyma resection significantly impacts long-term quality of life in patients with non-small cell lung cancer. Chest. 2009;135:322-9.

42. Garcia DO, Thomson CA. Physical activity and cancer survivorship. Nutr Clin Pract. 2014:29:768-79.

43. McNeely ML, Campbell KL, Rowe BH, Klassen TP, Mackey JR, Courneya KS. Effects of exercise on breast cancer patients and survivors: a systematic review and meta-analysis. Can Med Assoc J. 2006;175:34-41.

44. Ha D, Mazzone PJ, Ries AL, Malhotra A, Fuster M. The utility of exercise testing in patients with lung cancer. J Thorac Oncol. 2016;11(9):1397-410.

45. Benzo R, Kelley GA, Recchi L. Complications of lung resection and exercise capacity: a meta-analysis. Respir Med. 2007;101:1790-7.

46. Loewen $\mathrm{GM}$, Watson D, Kohman L. Preoperative exercise $\mathrm{VO}_{2}$ measurement for lung resection candidates: results of Cancer and Leukemia Group B Protocol 9238. J Thorac Oncol. 2007;2:619-25.

47. Brunelli A, Belardinelli R, Refai M. Peak oxygen consumption during cardiopulmonary exercise test improves risk stratification in candidates to major lung resection. Chest. 2009:135(5):1260-7.

48. Jones LW, Watson D, Loewen G, et al. Peak oxygen consumption and long-term all-cause mortality in non-small cell lung cancer. Cancer. 2010;116:4825-532.

49. Lai JS, Wong MK, Lan C, Chong CK, Lien IN. Cardiorespiratory responses of Tai Chi Chuan practitioners and sedentary subjects during cycle ergometry. J Formos Med Assoc. 1993;92:894-9.

50. Lai JS, Lan C, Wong MK, Deng SH. Two-year trends in cardiorespiratory function among older Tai Chi Chuan practitioners and sedentary subjects. J Am Geriatr Soc. 1995;43:1222-7.

51. Liu J, Chen P, Wang R, Yuan Y, Wang X, Li C. Effect of Tai Chi on mononuclear cell functions in patients with non-small cell lung cancer. BMC Complement Altern Med. 2015;15:3. doi:10.1186/s12906-015-0517-7.

52. Wang R, Liu J, Chen P, Yu D. Regular tai chi exercise decreases the percentage of type 2 cytokine-producing cells in postsurgical non-small cell lung cancer survivors. Cancer Nurs. 2013;36:27-34.

\section{Submit your next manuscript to BioMed Central and we will help you at every step:}

- We accept pre-submission inquiries

- Our selector tool helps you to find the most relevant journal

- We provide round the clock customer support

- Convenient online submission

- Thorough peer review

- Inclusion in PubMed and all major indexing services

- Maximum visibility for your research

Submit your manuscript at www.biomedcentral.com/submit

) Biomed Central 